\title{
Comunicación lineal, dinámica y productiva en restaurantes: análisis de noticias antes y durante la CovID-19 en México y España
}

Linear, dynamic and productive communication in restaurants: news analysis before and during CovID-19 in Mexico and Spain

María Dolores Lozano Gutiérrez ${ }^{*}$

ISU Universidad

25 Sur núm. 702, Col. La Paz, C.P. 72160, Puebla,

Puebla, México

Editor: Rogelio del Prado Flores

Fecha de recepción: 9 de julio de 2020

Fecha de aceptación: 22 de julio de 2020 maria.lozanog@anahuac.mx https://orcid.org/0000-0002-7451-2346

\section{RESUMEN}

El propósito de este artículo es comparar el nivel de comunicación lineal, dinámica y productiva antes y durante la CoVID-19 en noticias de México y España con las palabras restaurante y comunicación en la plataforma Google, de enero a mayo 2020, para analizar la prioridad estratégica a la que se enfocan sus esfuerzos comunicativos. El análisis versa sobre la categorización de las noticias como comunicación de tipo lineal, dinámica o productiva a partir de la Teoría de la Comunicación Productiva de Abraham Nosnik (2001, 2012). Los resultados muestran una mayor proporción de comunicación lineal antes de la CoVID-19 y un incremento en la comunicación productiva durante la COVID-19 tanto en México como en España. Antes de la COVID-19 los temas de comunicación eran distintos en ambos países mientras que durante la COVID-19 se muestra una mayor coincidencia en los temas de comunicación.

Palabras clave: comunicación productiva, hospitalidad, empresas de servicios, CoviD-19, confianza.

* Profesor de ISU Universidad. Doctorante del doctorado en Investigación de la Comunicación en la Facultad de Comunicación de la Universidad Anáhuac México. Maestra en Administración de Tecnologías de la información y licenciada y maestra en Mercadotecnia. Ha ejercido por más de diez años en las áreas de investigación de mercados, desarrollo de producto, promoción, ventas y relaciones públicas. 


\begin{abstract}
The purpose of this article is to compare the level of linear, dynamic and productive communication before and during COVID-19 in news in Mexico and Spain with the words restaurant and communication on the Google platform from January to May 2020 to analyze the strategic priority to which their communication efforts are focused. The analysis deals with the categorization of news as linear, dynamic or productive communication based on Productive Communication Theory by Abraham Nosnik $(2001,2012)$. The results show a higher proportion of linear communication before COVID-19 and an increase in productive communication during COVID-19 in both Mexico and Spain. Before CovID-19, communication issues were different in both countries, while after COVID-19, there was greater agreement on communication issues.
\end{abstract}

Keywords: productive communication, hospitality, service companies, COVID-19, trust.

\title{
INTRODUCCIÓN
}

nte la pandemia de la covid-19 uno de los sectores más afectados es la indus-
tria restaurantera y su cadena de valor (Cepal, 2020) que incluye los proveedores que con sus bienes y servicios participan en el ofrecimiento de un servicio. El impacto de la Covid-19 va más allá de un cierre temporal de los restaurantes. Por un lado, la necesidad de un distanciamiento social al interior de los restaurantes obliga a los dueños de los restaurantes a modificar su modelo de negocio de modo que pueda ser rentable atendiendo a un número reducido de personas. Por otro lado, la nueva normalidad exige el aseguramiento de las condiciones de sanidad e higiene al interior del restaurante. Ambas situaciones demandan una comunicación que transmita información (comunicación lineal), la distribuya (comunicación dinámica) y haga uso de ella para crear un ambiente propicio que lleve al sistema al logro de sus propósitos (comunicación productiva).

Al realizar la búsqueda de los conceptos comunicación, restaurantes y CoVID-19 en Google Scholar se presentan 526 resultados, los artículos versan sobre el tema de cierre de restaurantes, pérdidas y afectación del sector por lo que partiendo de que la situación de la CoviD-19 es una situación actual se optó por revisar las 
noticias de la plataforma Google con la finalidad de tener un panorama de lo que está surgiendo en torno al tema de comunicación y restaurantes.

\section{MARCO TEÓRICO Y CONTEXTUAL}

El fenómeno covid-19 desde el ámbito de los restaurantes como empresas de servicio tiene varios enfoques de estudio. El primero está relacionado con que las empresas de servicio son un foco de contagio por la presencia de personas. El segundo tiene que ver con el tema financiero donde en el corto plazo al existir un menor consumo, las empresas han buscado formas de reducir gastos recurriendo al despido de personal que a su vez contribuye a contraer aún más la economía (Deloitte, 2020). El tercero, atendiendo a las medidas de sana distancia plantean una reingeniería del modelo de negocio de la industria restaurantera que requiere no basarse más en temas de economías de escala, de alta ocupación o rapidez de rotación de las mesas además de considerar la inclusión de estrategias omnicanal, varios canales de venta, para hacer llegar sus productos a los clientes que plantea el agregar intermediarios y nuevos socios comerciales con su respectiva retribución, lo que afecta aún más el margen de utilidad del restaurante.

Los restaurantes son empresas de servicios que tienen como característica un alto contacto entre personal y consumidor. Remitiéndose al proceso de atención al cliente en un restaurante existen múltiples contactos interpersonales desde el momento en que llega al restaurante por parte del valet parking, personal de seguridad o hostess; durante toda su estancia es asistido por uno o varios meseros; ocasionalmente también puede ser abordado por el gerente; además de los posibles encuentros con otros clientes.

Las formas de contagio de la Covid-19 suceden cuando se tiene contacto con una persona enferma o bien cuando las gotículas respiratorias del enfermo se quedan en superficies u objetos (oMs, 2020). Las empresas de servicio se caracterizan por la convivencia de personas tanto del personal que atiende como de los mismos clientes entre sí. Considerando que la covid-19 se hospeda en las personas y que su contagio tiene relación con el contacto con otras personas, es entendible que las empresas de servicios sean los sectores más afectados por las medidas de distanciamiento social y cuarentena (Cepal, 2020). En específico se señala a los restaurantes como uno de los sectores más afectados a raíz de ls Covid-19 (Coneval, 2020). 
El impacto de la covid-19 en España se pronostica en una disminución del PIB de 7.7\% mientras que en México se estima una disminución del 6.5\% del PIB en el año 2020. En España se maneja en la terminología de derecho laboral, en la Clasificación Nacional de Actividades Económicas y por el Instituto Nacional de Estadística el término hostelería para aglutinar el alojamiento u hospitalidad y la restauración. La organización que representa a los restaurantes, cafeterías y pubs es la Confederación Empresarial de Hostelería de España. El sector de los restaurantes emplea a 1.3 millones de personas y aportan el 4.7 del PIB nacional (CEHE, 2019). En contraste en México los restaurantes generan 1.7 millones de empleos directos (Secretaría de Turismo, 2018) y aportan el 2\% del PIB nacional (America-Retail, 2019). El gasto medio del consumidor en restaurantes en España es de 4.88 euros por visita estimando como número de visitas promedio 159 al año (KPMG, 2018). En México, en los hogares se destina alrededor de 35\% del gasto cotidiano en alimentos y bebidas. Del porcentaje anterior donde el $23 \%$ se destina al consumo de alimentos fuera del hogar (INEGI, 2019).

España se autodescribe como una potencia gastronómica reconociendo su biodiversidad; la existencia de 206 restaurantes que ostentan estrella Michelín, el máximo reconocimiento gastronómico del mundo; 7 restaurantes ubicados en la lista The World's 50 Best Restaurants 2018; país cuna de movimientos gastronómicos como la Nueva Cocina Vasca que aglutina a destacados chefs a nivel internacional y como referente del turismo gastronómico (KPMG, 2019). México, por su parte, cuenta con la denominación de la gastronomía mexicana como Patrimonio Cultural Inmaterial de la Humanidad (Unesco, 2010) y cuenta con una biodiversidad ancestral (Iturriaga, 2018). En México como tal no existe un restaurante que tenga el reconocimiento estrella Michelin, no obstante, existen tres chefs mexicanos con restaurantes en Madrid, Barcelona y Chicago que sí ostentan estrella Michelín (Gourmet de México, 2020). Respecto al listado The World's 50 Best Restaurants 2018 dos restaurantes en México figuran en esta prestigiada lista (The Worlds 50 best, 2020).

Los restaurantes en España muestran una disminución del tránsito de personas del $24 \%$ en los meses de mayo y junio de 2020 mientras que en México es de 56\% (Google Mobility, 2020). En España la facturación anual del sector de la hostelería podría disminuir hasta un $40 \%$ y el empleo podría verse impactado hasta en una disminución de $25 \%$ (EY, 2020), expertos hablan de la desaparición de $20 \%$ de los restaurantes existentes antes de la covid-19 (Marín, 2020). En México cifras de la Canirac revelan que a partir de la covid-19 el 80\% de los res- 
taurantes están cerrados; $15 \%$ han adaptado su servicio a domicilio y para llevar; la caída de la demanda ha sido de un 85\%; 30000 restaurantes han cerrado en forma definitiva y existe el riesgo de pérdida de empleo para 300000 familias (Fernández, 2020).

El sector restaurantero está muy fragmentado y constituido por pequeños negocios, opera con márgenes de utilidad muy bajos (6\%), tiene niveles de capitalización bajos y poca liquidez que les permite sobrellevar los gastos operativos solo en periodos cortos de tiempo ( $50 \%$ de los negocios apenas aguantan un mes) (EY, 2020). Por todo lo anterior, Deloitte (2020) pronostica que el sector restaurantero será el último sector que restablezca su operatividad y consumo regular en marzo de 2021.

Además, la menor demanda de servicios en los restaurantes repercute en toda la cadena de proveeduría tanto de productos como de servicios. Por mencionar, algunos de los sectores más dañados son los empleados de los restaurantes pertenecientes a la población con menos ingresos económicos (Deloitte, 2020) que viven al día, los meseros por ejemplo su mayor ingreso está representado por las propinas que reciben; la industria cafetalera, la industria vinícola, industria panadera, industria cárnica, industria pesquera, industria de los lácteos e industria agroalimentaria por mencionar algunas. Sumado a lo anterior están los gastos fijos a los que incurre un restaurante como renta, luz, agua e internet adicional a las pérdidas derivadas de la materia prima que tenían en habían adquirido para su posterior uso (Makro, 2020).

Según Deloitte (2020) las estrategias de comunicación de los restaurantes varían en función del tipo de restaurante. Los restaurantes pymes no están llevando acciones de comunicación, los restaurantes de cadena cuentan con una estrategia planificada institucionalmente, mientras que los restaurantes insignia realizan acciones de responsabilidad social en apoyo al personal médico y comunidades en condiciones de pobreza alimentaria, mientras que los restaurantes insignia se centran en acciones de comunicación y mercadotecnia durante la contingencia hacia sus audiencias y algunos realizan acciones de responsabilidad social.

El escenario antes descrito plantea que los restaurantes tienen una falta de liquidez, han perdido a su factor humano y se enfrentan a la necesidad de ganar la confianza del cliente para que acuda nuevamente a los restaurantes. En cuestión de liquidez, los restaurantes se han enfocado en las ventas de sus alimentos a través plataformas digitales. En el mediano plazo, el sector restaurantero tiene el reto de reinventarse en lugar de volver a sus modelos de ocupación de espacio 
como fuente de rentabilidad como en el pasado. Comunicacionalmente hablando, los restaurantes requieren ser congruentes en que su operación comunique que ir a un restaurante es seguro sanitariamente (Deloitte, 2020). En términos comunicativos, lo anterior ha significado un cambio en las prioridades de comunicación que los restaurantes tienen, como se verá en la sección de resultados donde se presentan las estrategias de comunicación utilizadas por los restaurantes en los primeros cinco meses del año 2020.

Dado que el ámbito de las noticias de comunicación y restaurantes que se analizan son predominantemente latinoamericanos, se ha seleccionado la Teoría de la Comunicación Productiva de Abraham Nosnik, investigador mexicano. A continuación, se presentan los tres niveles de comunicación propuestos por este autor que serán el referente por considerar para ubicar las noticias analizadas.

La Teoría de la Comunicación Productiva parte del concepto de retroalimentación como detonador de procesos creativos y de innovación de la organización, así como de la necesidad de las organizaciones de estar en el paradigma equilibrio cambio en un contexto caracterizado por el advenimiento de tecnologías flexibles y convergentes. Desde el concepto de Nosnik $(2001,2012)$ y bajo un fundamento interaccional sistémico (Watzlawick, Bavelas, \& Jackson, 1991) donde el todo es mayor que las partes, es necesario partir del establecimiento de propósitos del sistema como elementos guía de la organización. Una vez enmarcado lo anterior se está en posibilidad de abordar las tres miradas que Nosnik establece de los esfuerzos comunicativos de una organización.

Bajo el paradigma mecanicista de Claude E. Shanon y Warren Weaver (1948) la comunicación lineal (Nosnik, 20 01, 2012) establece como propósito fundamental de la organización en el ámbito comunicativo la función de informar. Esta perspectiva es propia de organizaciones centradas en ellas mismas situando al resto de los actores implicados en la comunicación en meros receptores de información. Las limitantes de este tipo de comunicación radican en que su enfoque contempla solo el aspecto lingüístico centrándose únicamente en la comprensión de un mensaje dado. La comunicación lineal es por tanto unidireccional y deja de lado la riqueza de la interacción remitiendo al otro a ser una audiencia pasiva. El sustento teórico de la comunicación lineal está en la sintaxis centrada en los flujos de información en la Teoría de la información de Shanon y Weaver (1948) (Nosnik, 2012).

El centro de la comunicación lineal es la calidad de la información aludiendo al principio de ser íntegro, ser veraz, es decir a la responsabilidad del emisor, así 
como en la objetividad de la información. El principio de integridad alude a la veracidad en la producción y difusión de la información, es decir a la responsabilidad del emisor, así como en la objetividad de la información. El principio descrito se exceptúa en los casos de confidencialidad donde se entiende un interés legítimo a faltar a la verdad atendiendo al genuino bienestar de un tercero (Nosnik, 2001).

Con un sustento semántico, el nivel dinámico alude a los modelos complejos caracterizados por la presencia del factor humano. Este segundo nivel de comunicación tiene su foco en los receptores, entes complejos, dada las influencias a las que están expuestos, que interpretan los mensajes. Se trata de una comunicación bidireccional orientada al entendimiento y acuerdo entre las partes basadas en la retroalimentación. Remite a un proceso de comunicación empática. Este nivel remite al principio de ser integral, es decir, cumplir con todos los públicos (Nosnik, 2012).

El principio de ser integral se enfoca a la reputación, credibilidad, coherencia, confianza, cumplimiento con un enfoque hacia la responsabilidad social organizacional (RSO). Bajo este principio se propone a la comunicación dinámica, partiendo de que naturalmente se desea tener una imagen positiva que está conformada tanto de información objetiva como de percepciones, es menester atender las necesidades de los públicos a los que se dirige la comunicación de modo que se contribuya con la comunicación generada a la consecución de sus objetivos (Nosnik, 2001).

Bajo el paradigma pragmático, el nivel productivo se centra en el sistema donde se intercambia la información enfatizando el uso inteligente de los mensajes para cumplir el propósito del sistema. Se concibe como detonador del proceso productivo al receptor como referente de necesidades y expectativas de información. Este tercer nivel de comunicación, según Nosnik $(2001,2012)$, es el más avanzado dado que se enfoca en el cumplimiento y reinvención del sistema, es decir, a la comunicación integrada que considera todas las partes del sistema para la consecución del propósito general de la organización y la satisfacción interpersonal de los componentes o grupos que integran el sistema. La capacidad de la comunicación productiva versa sobre alinear los elementos de la organización hacia el cumplimiento de los propósitos de la organización como sistema, así como la congruencia. Para la comunicación productiva la credibilidad de los mensajes, actitudes y comportamientos se mide en función de la congruencia con los fines, medios, reglas y recursos del sistema lo que incluso lleva a cuestionar la autoridad de un individuo, un grupo o una organización (2012). 
En la comunicación integrada a la productividad, la comunicación es concebida como soporte, como plataforma para lograr la efectividad y la competencia del sistema fomentando la reinvención y permanencia de la organización inserta en un ambiente competitivo y cambiante. Desde esta concepción, al interior de la organización la comunicación es facilitadora de la calidad, del servicio, de la eficiencia, de los propósitos claros; al exterior de procesos, provee de enfoque a largo plazo para el establecimiento de propósitos, aporta eficiencia de recursos en la organización coadyuvando a tener un enfoque sustentable en la comunidad donde se sitúa la organización. Así el procesamiento de la información y la respuesta por parte del receptor completa el círculo de la comunicación destacando que los participantes en la comunicación son parte de un sistema donde el conjunto es más que las partes aisladas (Nosnik, 2001).

Enumerando las preguntas de Lasswell (1948) se tiene que la pregunta quién, enfocada en el emisor, así como la pregunta a quién, remiten a un enfoque lineal; la pregunta qué efecto se asocia a una gestión dinámica dado que considera el tema de la retroalimentación. Finalmente, la comunicación productiva asume tres responsabilidades con respecto a la información: considerar las necesidades y expectativas de los receptores (lineal), distribuir la información a quién la requiera (dinámica), buscar el bien común (productiva).

Internet se ha convertido mediante las redes sociales en uno de los principales informadores (Sánchez de la Nieta \& Fuente, 2020) en este ámbito se hace necesaria la gestión de la comunicación digital integral para las organizaciones, Rebeca Arévalo y María Antonieta Rebeil (2016) proponen un modelo para que la organización haga uso efectivo de los medios con los siguientes elementos: presencia, impacto, proximidad, colaboración, ética, RSO como puede apreciarse los dos primeros elementos son de tipo lineal, mientras que los restantes se relacionan con la comunicación dinámica antes enunciada.

\section{METODOLOGÍA}

Se realizó un análisis de las noticias en español relacionadas con el ámbito de la comunicación y los restaurantes en la plataforma de Google antes y durante la Covid-19. Las noticias revisadas son del periodo enero-mayo de 2020. El recuento de las noticias fue de 156: España (76) y México (78). Se identifica como fecha de referencia para este análisis antes y durante la CoviD-19, la fecha en la 
que los restaurantes en México comienzan con protocolos para hacer frente a la situación generada por la coviD-19 que fue el 12 de marzo del 2020. En España no se reportan noticias en la plataforma de Google que manifiesten el inicio de protocolos en restaurantes antes del 12 de marzo de 2020.

\section{RESULTADOS}

La mezcla de tipo de comunicación presente en las noticias relacionadas con restaurantes en España es: $41 \%$ de noticias bajo la categoría comunicación lineal; $33 \%$ de noticias con comunicación de tipo dinámica y $26 \%$ de noticias de comunicación tipo productiva. En México se tienen $64 \%$ de noticias bajo la comunicación lineal, $15 \%$ de noticias bajo la comunicación y dinámica y $21 \%$ de noticias de comunicación de tipo productiva (Ver Figura 1).

FIGURA 1. TIPO DE COMUNICACIÓN UTILIZADA EN LAS NOTICIAS

ANALIZADAS EN ESPAÑA Y MÉXICO (ENERO A MAYO DE 2020)

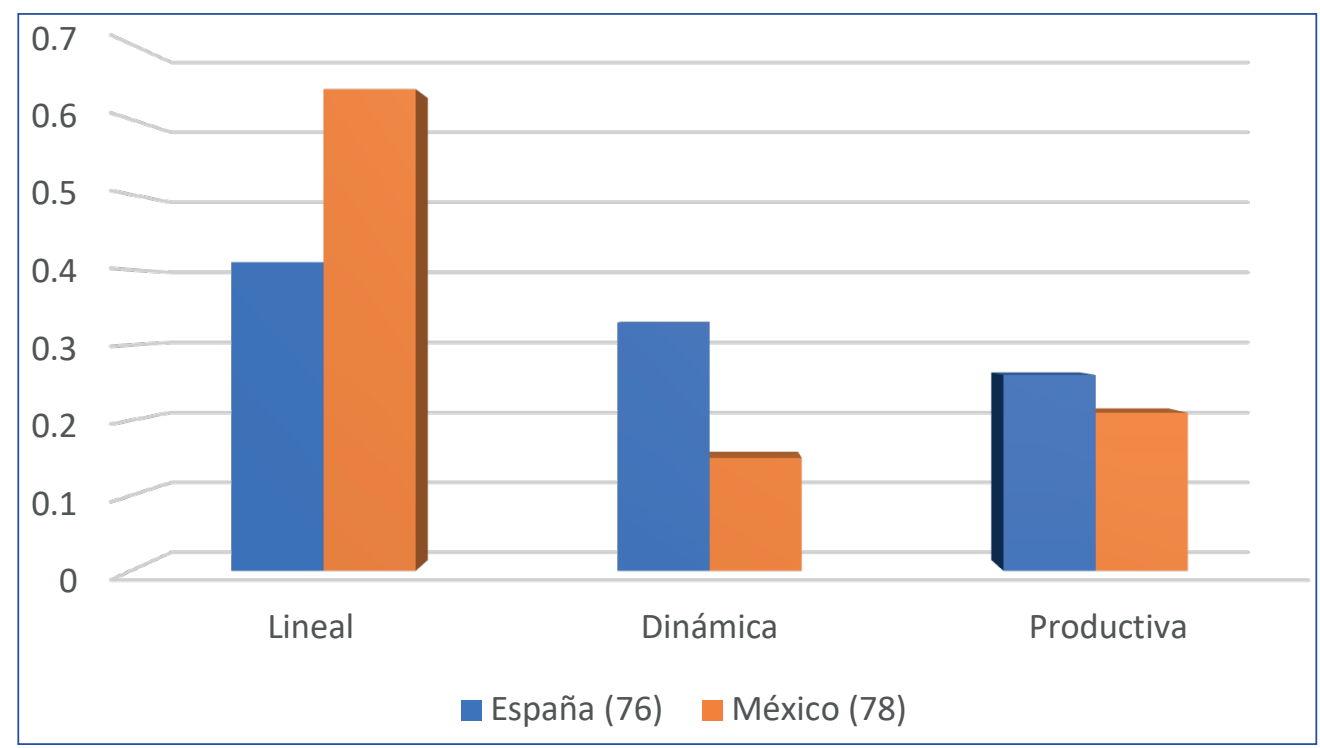

FUENTE: ELABORACIÓN PROPIA.

Haciendo un comparativo de las noticias antes y durante la covid-19 se advierte un incremento en cuanto a la generación de noticias de tipo productivo 
a partir de la covid-19. Antes de al covid-19 en el continente europeo el 55\% de noticas eran enfocadas a la comunicación lineal, 33\% a la comunicación dinámica y $13 \%$ a la comunicación productiva. Durante la covid-19 el 37\% de noticas eran enfocadas a la comunicación lineal, $34 \%$ a la comunicación dinámica y $29 \%$ a la comunicación productiva (Ver Figura 2).

FIGURA 2. TIPO DE COMUNICACIÓN PRESENTE EN LAS NOTICIAS

ANALIZADAS EN ESPAÑA ANTES Y DURANTE LA COVID-19

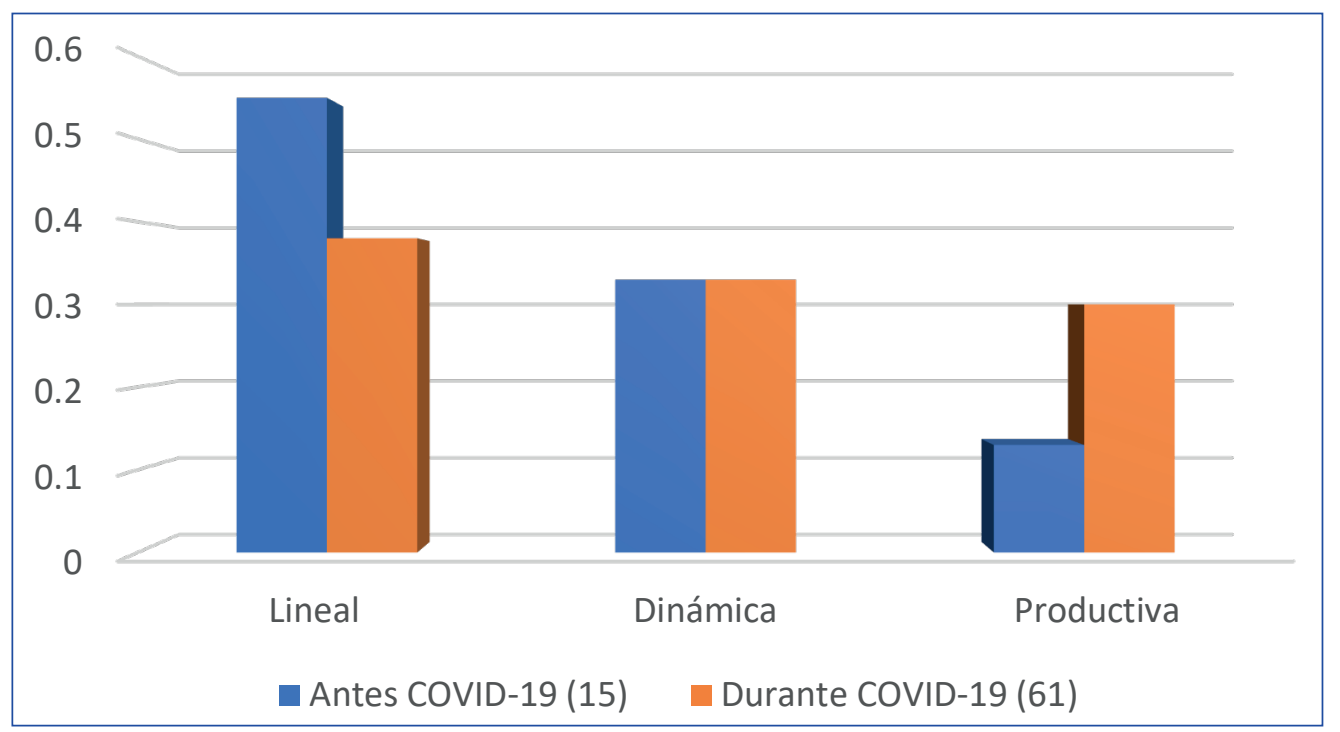

FUENTE: ELABORACIÓN PROPIA.

Por su parte, en México antes de la coviD-19, el 83\% de noticas eran enfocadas a la comunicación lineal, $17 \%$ a la comunicación dinámica no se encontraron noticias con comunicación productiva. Durante la covid-19 el 58\% de noticas eran enfocadas a la comunicación lineal, $15 \%$ a la comunicación dinámica y $27 \%$ a la comunicación productiva (Ver Figura 3). 
FIGURA 3. TIPO DE COMUNICACIÓN PRESENTE EN LAS NOTICIAS ANALIZADAS EN MÉXICO ANTES Y DURANTE LA COVID-19

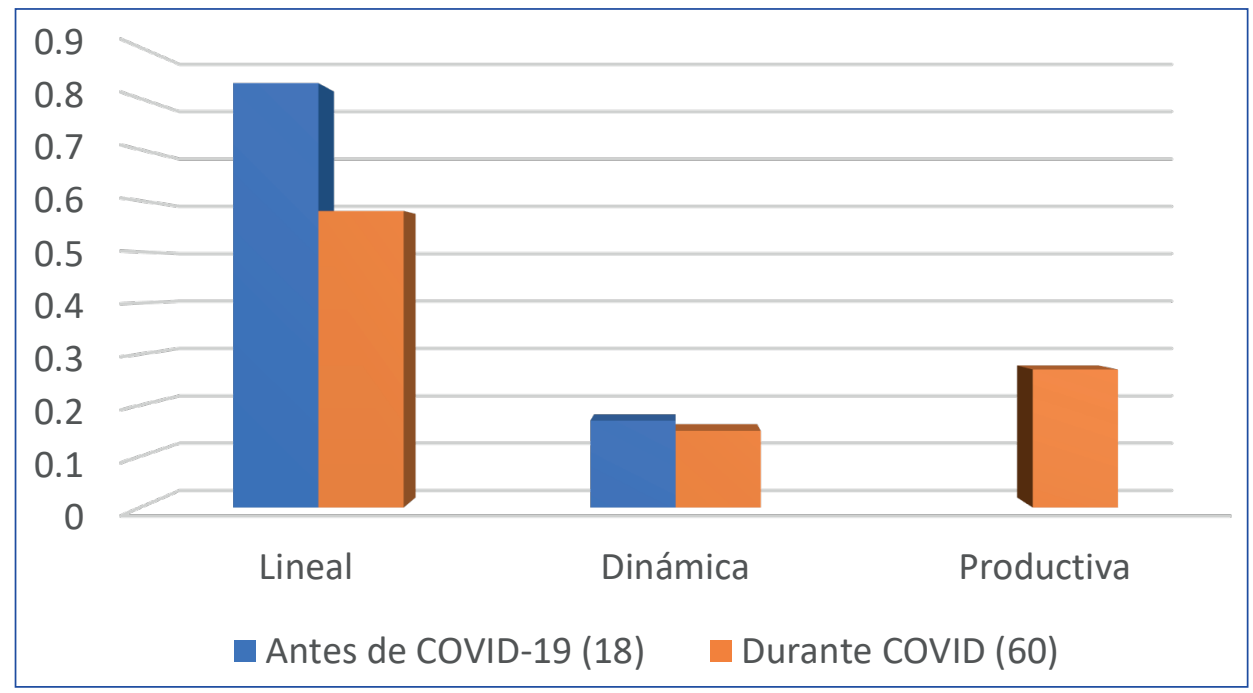

FUENTE: ELABORACIÓN PROPIA.

Antes de la Covid-19 en España más de una cuarta parte de las noticias analizadas abordan el tema de la digitalización de los restaurantes (27\%); iniciativas de Responsabilidad Social (13\%); las redes sociales como herramienta de comunicación de los restaurantes (13\%); reconocimientos en el ámbito gastronómico (13\%); cierre de restaurantes (13\%); noticias aisladas en cuanto a temas de pérdidas económicas de un restaurante, comunicación especializada en el sector ofrecida por una universidad. Por su parte, en México los temas más tratados en las noticias analizadas fueron noticias particulares a restaurantes específicos (28\%), es decir, la temática estaba regida por lo que surgiera de novedoso; el tema de la inseguridad era el tema recurrente (11\%); los reconocimientos a restaurantes o talentos gastronómicos (11\%); el cumplimiento a normativas por parte de los restaurantes (11\%); los avances en temas de digitalización en torno al sector restaurantero (11\%); noticias con solo una mención incluyen el 14 de febrero como temporalidad de venta, una propuesta de servicio inclusivo, una iniciativa de Responsabilidad Social por parte de un restaurante, el avance de una plataforma de servicios de entrega y la efectividad de la comunicación generacional en los restaurantes (Ver Figura 4). 
FIGURA 4. TEMAS ABORDADOS EN LAS NOTICIAS DE ESPAÑA Y MÉXICO ANTES DE LA COVID-19

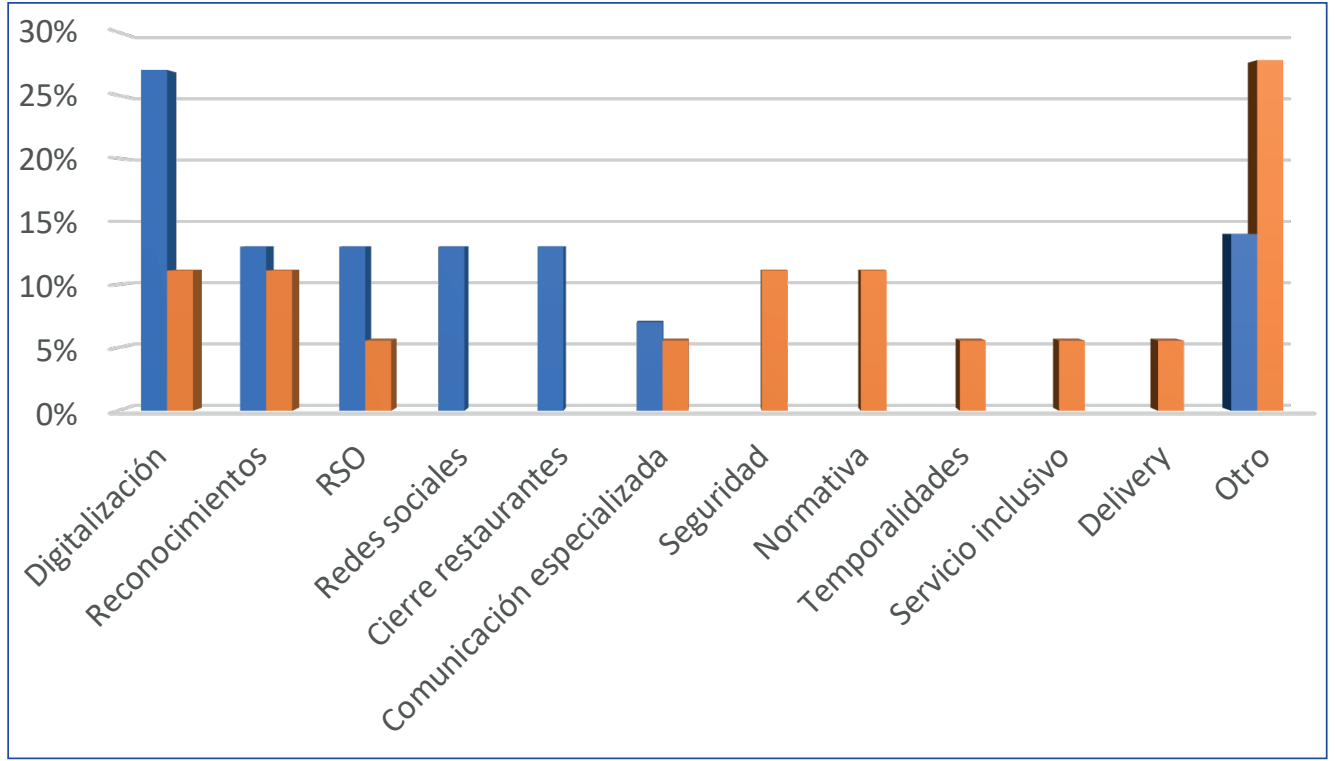

FUENTE: ELABORACIÓN PROPIA.

En España antes de la covid-19 la comunicación lineal de los restaurantes estaba centrada básicamente en dos temas. Uno, el tema de la digitalización de los restaurantes con el lanzamiento de aplicaciones para uso dentro del restaurante, asistentes virtuales y el empleo de herramientas digitales para llegar a nuevos mercados. De manera más aislada las noticias restantes informan respecto a talentos gastronómicos de centros culinarios reconocidos; lanzamiento de algún producto alimenticio o de algún destino gastronómico; dan a conocer la propuesta de posicionamiento de marca de una franquicia de restaurantes orientada a ser empática con los clientes y con la responsabilidad; informan sobre el cierre de un restaurante a inicios de año; así como las problemáticas de un restaurante que a pesar de sus acciones de responsabilidad social organizacional se ha visto obligado a cerrar. La comunicación dinámica en España aborda el impulso a la comunicación especializada en restauración y bodegas vinícolas; el pronunciamiento de franquiciatarios que demandan mayores apoyos de la marca franquicia y los beneficios del uso de las redes sociales por parte de los restaurantes en términos de tener una comunicación más personal con los clientes. Finalmente, la comunicación productiva se orienta a destacar la aplicación desarrollada por empren- 
dedores españoles que permiten al usuario hacer sus pedidos incluso antes de llegar al restaurante y de pagar a través de la plataforma así de otra plataforma de gestión de pedidos de los clientes vinculando al área de salón comedor, cocina, gerente e incluso a los clientes.

En México, antes de la covid-19 las noticias en el ámbito de los temas comunicación y restaurantes se centraban en el tema de seguridad que originaba el cierre de restaurantes, así como en informar acerca de incidentes violentos en restaurantes. Otro tema que abordan las noticias era la digitalización destacando el uso de las plataformas de delivery en Ciudad de México y Guadalajara. En otra categoría de noticias están las que impulsan el turismo gastronómico, la difusión de la gastronomía mediante publicaciones, el nombramiento de restaurantes dentro de una guía de nombramientos gastronómicos. En torno a la comunicación dinámica solo se identifican tres noticias bajo este rubro de comunicación. La primera surge como una respuesta empática de los restaurantes ante la situación de inseguridad que lanzan una iniciativa de los restaurantes en Los Mochis en ofrecer el restaurante como espacio seguro para las mujeres en caso de peligro. La segunda se aboca a despertar la conciencia del ámbito restaurantero respecto a la necesidad de una comunicación distinta para las personas con discapacidad visual, para ofrecerles un servicio inclusivo, por lo que se ofrecen talleres para sensibilizar a los restaurantes. La tercera noticia destaca la necesidad de entender las diferencias generacionales que al interior de los restaurantes se dan de modo para favorecer el uso de las nuevas tecnologías de información y comunicación para comunicarse con los empleados.

Durante la Covid-19 en España se identifican 61 noticias que tienen como temas preponderantes el tema de los cambios que los restaurantes requieren realizar en cuanto a su modelo de operación y de negocio para abrir de nuevo sus puertas (16\%); las iniciativas que gobierno, medios, sociedad, socios de valor y otros organismos han propiciado para apoyar a los restaurantes ante la situación por la que los restaurantes atraviesan (15\%); los protocolos que los restaurantes requieren cumplir de acuerdo con la normativa definida por las autoridades (13\%); la modalidad de entrega a domicilio y uso de plataformas a las que los restaurantes se han sumado para continuar operando (13\%); el uso de redes sociales como medio para continuar en comunicación con los comensales $(10 \%)$; las pérdidas económicas de los restaurantes derivadas de la covid-19 (7\%); la reapertura de los restaurantes (7\%); las posibles acciones que los restaurantes que podrían llevar a cabo en esta etapa de cuarentena al interior de la organiza- 
ción (7\%); la normativa que las autoridades han generado a partir de la situación de la covid-19 relacionada con el ámbito de los restaurantes (5\%); esfuerzos en temas de digitalización por parte de los restaurantes (3\%).

En México de las 60 noticias identificadas durante la covid-19 casi una cuarta parte de ellas (23\%) se concentran en los protocolos que los restaurantes requieren cumplir derivada de la normativa establecida. Cabe destacar que a lo largo del país se carece de uniformidad en cuanto a las medidas establecidas, así mientras en algunos estados los restaurantes aún continúan abiertos en otros estados están cerrados. Aproximadamente una quinta parte de las noticias (18\%) dan cuenta de las iniciativas que se han puesto en marcha para apoyar al rubro de los restaurantes. Dentro de estas iniciativas destacan el tema del delivery como opción para mantenerse en operación, ocupa el 13\% de la temática de las noticias; $13 \%$ están enfocadas al cierre de restaurantes, ya sea parcial o definitivamente; $7 \%$ se enfoca a los cambios operativos y de modelo negocio que los restaurantes requieren llevar a cabo para permanecer y continuar siendo rentables; $7 \%$ aborda el tema de las pérdidas económicas de los restaurantes a partir de la Covid-19; $7 \%$ se refiere a la celebración del 10 de mayo, Día de las madres, temporalidad que en tiempos normales es una importante fecha de venta en los restaurantes y la manera en que ante la cuarentena los restaurantes han pensado creativamente en ofrecer alternativas de consumo a los comensales; $5 \%$ comenta las violaciones a la normativa establecida en que los restaurantes han incurrido en esta cuarentena; $3 \%$ destaca las acciones de seguridad que ante el cierre de los restaurantes se requieren considerar para evitar atracos en los restaurantes; finalmente, noticias individuales abordan una campaña de un restaurante y otra noticia, las acciones que un restaurante lleva a cabo en estos tiempos de no actividad al público por parte de los restaurantes (Ver Figura 5).

Respecto a la categorización de las noticias en cuanto a comunicación lineal, dinámica y productiva básicamente, tanto en México como en España, la comunicación lineal se utiliza para informar de los cierres de restaurantes, de las pérdidas económicas generadas, los protocolos necesarios para que los restaurantes vuelvan a operar en un futuro y el tema de delivery como elemento para hacer llegar los alimentos a los comensales. Como elementos distintivos, mientras en México se habla de temas relacionados con la seguridad de los restaurantes al estar cerrados, violaciones a las medidas establecidas para los restaurantes, con la celebración del Día de las madres estando cerrados los restaurantes; en España se da a conocer la posible reapertura de los restaurantes, los cambios necesarios 
tanto operativamente como estratégicamente para que los modelos de negocio de los restaurantes pueden volver a abrir sus puertas de una manera rentable, las iniciativas legislativas para apoyar a los restaurantes.

FIGURA 5. TEMAS ABORDADOS EN LAS NOTICIAS DE ESPAÑA Y MÉXICO DURANTE LA COVID-19

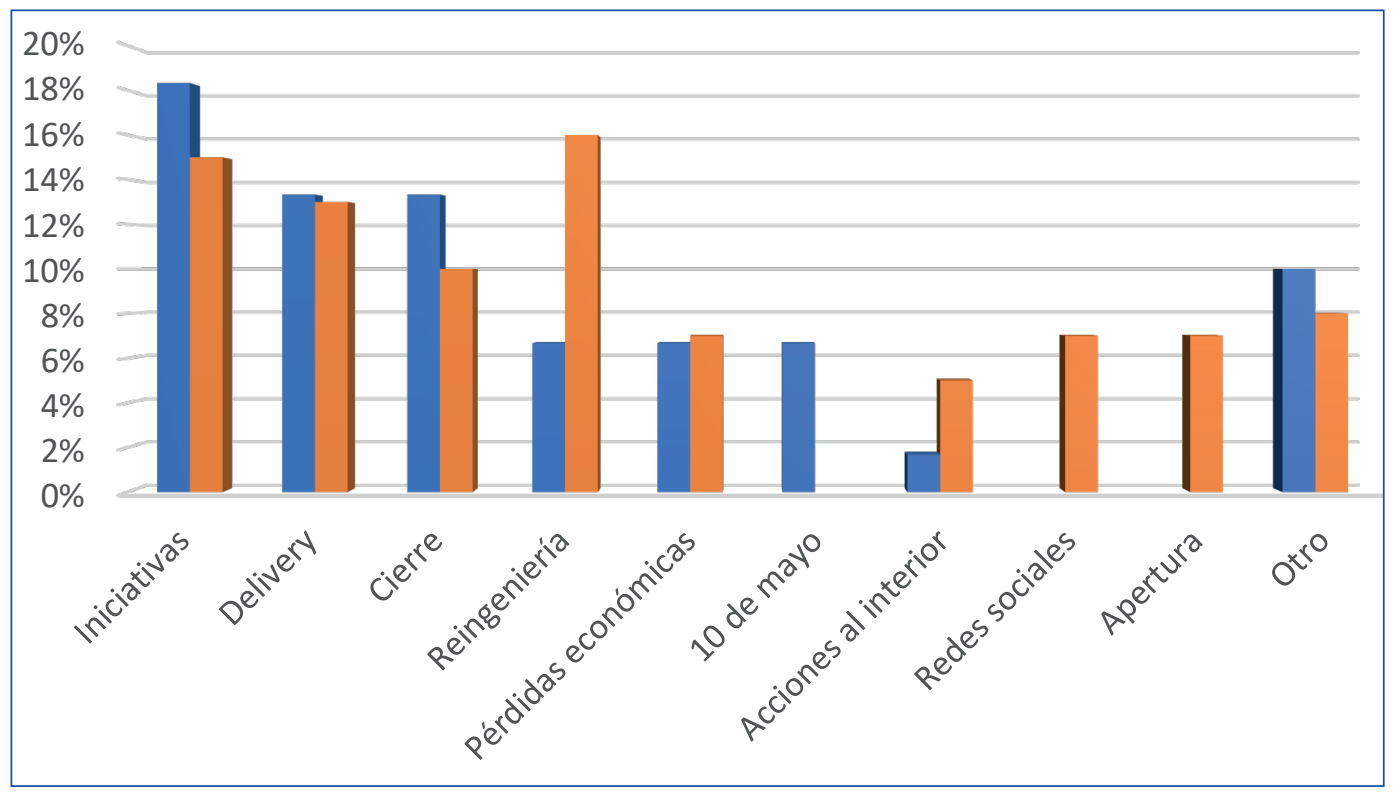

FUENTE: ELABORACIÓN PROPIA.

En relación con la comunicación dinámica en México se orienta al tema de cinco iniciativas dentro de las que destacan la iniciativa Ayuda a un restaurante, que en una plataforma digital de una empresa cervecera permite la compra de una tarjeta de regalo para uso posterior en restaurantes y la empresa duplica el valor de la tarjeta, el dinero recaudado se entrega a los restaurantes para pago de nómina y gastos; la iniciativa Academia de meseros lanzada por una empresa cervecera para capacitar en línea a los meseros de ciertas cadenas de restaurantes en temas de sus productos buscando que esta información les permita tener mejores propinas a la vez de promover los productos cerveceros con el comensal; una aplicación de WhatsApp para la aceptación de pedidos sin necesidad de presencia del personal de restaurantes. En España se maneja la iniciativa de la creación de un mapa con los restaurantes y tiendas de comida preparada para los trabajadores de servicios de la salud. 
Finalmente, en México la comunicación productiva incluye la iniciativa \#ComeCDMX que con la participación de medios, usuarios de redes sociales enfocados a gastronomía (foodies), la Secretaría de Turismo fomenta el consumo de alimentos y bebidas a domicilio, la propina y la compra a restaurantes locales en CDMX; la iniciativa \#SirviendoaMéxico de la Cámara de la Industria de Restaurantes y Alimentos Condimentados que llama a toda la cadena productiva, a productores de insumos, proveedores, caseros, bancos, clientes y gobierno, es una plataforma para difundir las estrategias que los restaurantes están llevando a cabo en este tiempo de contingencia (AMR, 2020); la iniciativa \#AquíNadieTruena impulsada por restauranteros del estado de Jalisco pidiendo a la población consumir lo local, apoyar a los pequeños y medianos negocios que siguen brindando sus servicios, fomenta la donación de alimentos a las personas que más lo necesitan en unión con el Banco de Alimentos (Excelsior, 2020) y el protocolo Mesa Segura que promueve la Canirac para ofrecer condiciones de sanitización e higiene así como de aforo y distanciamiento físico para brindar confianza a los comensales para hacer uso de los restaurantes (Canirac, 2020).

En España las noticias en línea con la comunicación productiva hablan de la oferta de la asesoría de organismos de gobierno, agencias de comunicación, cámaras a pymes incluidos los restaurantes sobre temas de comunicación; el apoyo de la sociedad va desde mensajes de aliento a los restaurantes; realización de auditorías digitales gratuitas a los restaurantes durante el periodo de inactividad; restaurantes que ofrecen de manera gratuita a los transportistas servicio de autoservicio de bebidas y alimentos donde ellos toman lo que requieren sin pagar y la iniciativa \#CocinadelRetorno, un festival gastronómico para impulsar la restauración de Málaga, Cádiz y Granada después de la covid-19.

\section{ANÁLISIS Y DISCUSIóN}

A partir de lo expuesto en el marco teórico y contextual, en el análisis de noticias durante la Covid-19 solo se encontró una noticia que habla de las pymes, lo que coincide con lo expuesto por Deloitte (2020) que asegura que los restaurantes pymes no están llevando a cabo acciones de comunicación. Respecto a la comunicación de los restaurantes de cadena orientada a la responsabilidad social (Deloitte, 2020) solo se halló una noticia que se trata de un grupo de restaurantes de España que están regalando comida al personal de servicios de salud. Respecto 
a los restaurantes insignia que disponen su comunicación a la mercadotecnia y a conectar con sus audiencias (Deloitte, 2020) se identifica la campaña \#CocinaDelRetorno que aglutina a prestigiados restaurantes de Málaga, Cádiz y Granada.

La predominancia de noticias durante la Covid-19 en torno al tema de los protocolos de operación a seguir por los restaurantes pone de relieve la prioridad de los restaurantes de comunicar que el restaurante es seguro (Deloitte, 2020). Además, los restaurantes se sitúan en el paradigma equilibrio cambio que Nosnik (2012) establece en su Teoría de la Comunicación Productiva. Por un lado, requieren tener un equilibrio entre sus necesidades organizacionales, las necesidades de sus colaboradores, las necesidades de sus proveedores y por supuesto las necesidades de sus comensales; por otro, requieren adaptar su manera de operar a la situación actual imperante.

Hablando de comunicación lineal, tanto en España como en México, antes de la CoviD-19 era el tipo de comunicación predominante siendo las audiencias meras receptoras de información (Nosnik, 2012). En relación con la comunicación dinámica, ante fenómenos complejos que incluyen el factor humano, como lo es la situación de la CoviD-19, fue necesario en un primer momento el entendimiento (Nosnik, 2012) de los restauranteros de cerrar sus establecimientos; posteriormente, para generar credibilidad y confianza (Nosnik, 2012) los restaurantes comenzaron a sumarse a la estrategia de delivery y promoción de sus servicios en redes sociales. Respecto a la comunicación productiva tanto en España como México se presentó un incremento en la comunicación de este tipo y una disminución de la comunicación lineal. En México incluso las noticias de comunicación productiva $(27 \%)$ sobrepasaron a la comunicación dinámica (15\%). La mayor parte de las iniciativas de comunicación productiva se generaron por actores de asociaciones, cámaras, Gobierno, empresas de la iniciativa privada, medios y sociedad, es decir, ajenos al sistema denominado restaurante. En México, el protocolo denominado Mesa Segura (Canirac, 2020) ejemplifica lo que Nosnik (2012) denomina cumplimiento y reinvención del sistema planteando un nuevo paradigma en la operación, estrategia y rentabilidad de los restaurantes que lleve a la mejora del sistema en su totalidad, así como la mejora de cada uno de los integrantes del sistema. Para la comunicación productiva la credibilidad de los mensajes, actitudes y comportamientos se mide en función de la congruencia del sistema (Nosnik, 2012). En el sistema denominado restaurante la congruencia será evaluada en la confianza que los comensales tengan en la operación y atención que se les brinde respetando los principios de sanidad, higiene y distanciamiento social siendo el reto 
mayor al que actualmente se enfrentan los restaurantes para ganarse la confianza de los clientes y lograr su retorno (Deloitte, 2020).

La comunicación es en estos momentos de cambio de paradigma un soporte, una plataforma para lograr la efectividad y permanencia de la organización en un ambiente complejo y cambiante (Nosnik, 20 01). Respecto al modelo de gestión de la comunicación digital integral para las organizaciones (Arévalo \& Rebeil, 2016) antes de la CoviD-19 se hace presente el elemento impacto presente en $13 \%$ de las noticias analizadas en España y $11 \%$ en México y el elemento RSO presente en 13\% de las noticias en España: después de la CoviD-19 el elemento RSO está presente en $15 \%$ de las noticias de España y 18\% en las noticias de México.

\section{CONCLUSIONES}

Al clasificar las noticias por tipo de comunicación: lineal, dinámica o productiva, a nivel total se observa una mayor predominancia de la comunicación de tipo lineal en las noticias pertenecientes a México. No obstante, al hacer el análisis en dos momentos, antes y durante la CoVID-19, se presenta una disminución de las noticias con comunicación de tipo lineal registrándose una mayor presencia de la comunicación dinámica y productiva. En el caso de España se presenta el mismo fenómeno, aunque en menor medida a comparación de México, es decir, desde antes de la coviD-19 la comunicación en Europa ya presentaba proporciones más equilibradas entre la comunicación lineal, dinámica y productiva. En México, en contraparte, existía una mayor concentración en las noticias con comunicación de tipo lineal por lo que pareciera que las situaciones adversas detonan los procesos de comunicación productiva orientados al bien común.

El análisis de contenido de las noticias muestra cómo antes de la COVID-19 en México no existía un tema rector de la comunicación en los restaurantes. En España sí existía una concentración de las noticias de los restaurantes hacia el tema de la digitalización además de existir esfuerzos de comunicación orientados a dar mayor visibilidad y diferenciación de los restaurantes bajo los temas del uso de las redes sociales, la Responsabilidad Social y los reconocimientos conseguidos por los restaurantes. En el caso de México existe una disgregación de temas que van desde la realidad del país en temas como la seguridad, la normativa, sin embargo, pareciera que el común denominador de las temáticas de las noticias es el día a día, excepto algunos esfuerzos aislados por destacarse bajo la bandera de 
la Responsabilidad Social o sumarse a la tendencia del uso de plataformas para entrega de alimentos.

Durante la CoviD-19 se presenta una mayor coincidencia en los temas abordados destacando cinco temas prioritarios que son los protocolos de operación de los restaurantes ante la COVID-19, las iniciativas ante la inactividad de los restaurantes, las estrategias de delivery, el cierre de los restaurantes y las pérdidas económicas derivadas de la pandemia por Covid-19.

Para quien esto suscribe, en el ámbito de la covid-19 la comunicación es facilitadora de la generación, implementación y control de procesos de convivencia organizacional y social que permitan a los restaurantes la consecución de sus propósitos en el nuevo ambiente sanitario.

Dentro de las limitaciones del presente estudio están que se excluyen aquellas noticias que no fueron consideradas por la plataforma Google durante el periodo de tiempo analizado. Finalmente, como futuras líneas de investigación se contempla el análisis de noticias en redes sociales, así como una metodología integral donde se contraste lo que dicen los medios, los usuarios y los restaurantes.

\section{REFERENCIAS}

Campos, M. A. (2005). Construcción de conocimiento. Universidad Nacional Autónoma de México.

Canirac. (2020, 13 de mayo). Protocolo de prevención CoviD-19: Mesa segura. https://canirac.org.mx/images/notas/files/Protocolo\%20Reapertura\%20-\%20 Mesa\%20Segura compressed.pdf

Cartulinas Flourescentes. (2020, 3 de junio). Anuncios flourescentes. https://www.instagram.com/cartulinasfluorescentes/?utm_source=ig_embed

Lego, E. (2020, 23 de marzo). Trabajo bajo protesta: taquilleras. https://twitter.com/enriquelego/status/1242124561317089280/photo/1

CEHE. (2019). Qué es hostelería de España. https://www.cehe.es/que-es-hosteleria-espana.html

CEPAL. (2020, 3 de abril). América Latina y el Caribe ante la pandemia del CoviD 19. Efectos económicos y sociales. https://repositorio.cepal.org/bitstream/handle/ 11362/ 45337/4/S2000264_es.pdf

CONCANACO. (2020, 11 de mayo). Mi tienda segura. https://www.concanaco.com.mx/ mi-tienda-segura/ 
Coneval. (2020). La política social en el contexto de la pandemia por el virus SARS-CoV-2 (Covid-19) en México. https://www.coneval.org.mx/Evaluacion/IEPSM/Documents/ Politica_Social_COVID-19.pdf

America-Retail. (2019, 6 de junio). México: Industria restaurantera impulsa economía mexicana. https://www.america-retail.com/mexico/mexico-industria-restauranteraimpulsa-economia-mexicana/

AMR. (2020). \#SirviendoaMéxico. http://www.amr.org.mx/noticias.phtml?id=3447\&categoria=NOTICIAS

Ansons, T. M. (2020, abril). (IPSOS, ed.) https://www.ipsos.com/sites/default/files/ct/publication/documents/2020-04/brand-rituals-low-touch-world_web.pdf

Arévalo, R., \& Rebeil, M. A. (2016). Presencia, interacción y responsabilidad social organizacional en las redes sociales digitales de organizaciones privadas en Iberoamércia. En R. Arévalo, \& M. A. Rebeil, Responsabilidad social en la comunicación digital organizacional. Tirant lo Blanch.

Augé, M. (1992). Los No lugares. Espacios del anonimato. Una antropología de la sobremodernidad. Gedisa.

Bacquerie, J. (2020). Animal MX. https://animal.mx/

Berger, P., \& Luckmann, T. (1966). La construcción social de la realidad. Amorrortu.

Deloitte. (2020). Webcast de la Industria Restaurantera. https://canirac.org.mx/images/ notas/files/Deloitte\%20Webcast_compressed.pdf

De Certeau, M. L. (1999). La invención de lo cotidiano 2. Habutar, cocinar (1a. ed.). Universidad Iberoamericana, Instituto Tecnológico y de Estudios Superiores de Occidente.

Excelsior. (2020,6 de abril). ¿Qué hay detrás y qué sigue del movimiento "Aquí nadie truena"? https://www.youtube.com/watch?time_continue=8\&v=5HGS2 mbkMpc\&feature= emb title\&fbclid=IwAR3NsBFRJLwPXMVCFwJ478SigBYaHtqGhBDGzuPLp6-eg8AJZC6CLdzYRTE

EY. (2020, abril). Impacto de COVID 19 en hostelería en España. https://www.ey.com/Publication/vwLUAssets/ey-bain-company-impacto-de-covid-19-en-hosteleria-en-espana/\$FILE/ey-bain-company-impacto-de-covid-19-en-hosteleria-en-espana.pdf

Flores, S. (2020). Letras. https://www.letras.com/chava-flores/1063177/

Fernández, F. (2020). Información y opiniones preparadas por CANIRAC. (Deloitte, entrevistador).

Glasersfeld, E. v. (2007). Key Works in Radical Constructivism. SensePublishers.

Google LLC. (2020). Google CoviD-19 Community Mobility Reports. https://www.google. com/: https://www.google.com/covid19/mobility/

Gourmet de México. (2020, 10 de enero). ¿Hay estrellas Michelin en México? https://gourmetdemexico.com.mx/comida-y-cultura/hay-estrellas-michelin-en-mexico/

Habermas, J. (1999). Teoría de la acción comunicativa II. Taurus. 
Hurtado, D. \& Aguirre, G. (2017). La colonia La Condesa en Ciudad de México: Conceptos y productores de lugar. Bitácora urbano territorial, 27(3).

INEGI. (2017). Características de los hogares. Instituto Nacional de Estadística y Geografía.

INEGI. (2019, 31 de julio). Resultados del ENIGH 2019. https://www.inegi.org.mx/contenidos/saladeprensa/boletines/2019/EstSociodemo/enigh2019_07.pdf

Instituto de Investigaciones Sociales. (2018, 16 de agosto). Narrativas de Categorías Sociales. Instituto de Investigaciones Sociales. https://es.slideshare.net/IIeXLatam/ iiexlatam-2018-heriberto-lpez

IPSOS Public Affairs. (2020, abril). What worries the world? https://www.ipsos.com/sites/ default/files/ct/news/documents/2020-04/whatworriesworld_april2020_global.pdf

IPSOS. (2020, 31 de enero). The IPSOS reputation council. https://www.ipsos.com/sites/ default/files/ct/publication/documents/2020-04/the-ipsos-reputation-council-report-april-2020_1.pdf

Iturriaga, J. N. (2018). Biodiversidad y diversidad cultural de México: una cocina históricamente nutritiva y sostenible. En U. México, El Origen y la evolución de la producción de alimentos y su impacto en los patrones de consumo (págs. 173-177). Unesco México.

Kotler, P. (2018). The Future of Marketing: An Interview with Philip Kotler, the "Father of Modern Marketing. (S. Shaw, entrevistador).

Kotler, P. (2020, 4 de junio ). The Saratosta Institute. https://sarasotainstitute.global/ the-consumer-in-the-age-of-coronavirus/

KPMG. (2019, enero). La gastronomía en la economía española. https://assets.kpmg/content/dam/kpmg/es/pdf/2019/01/gastronomia-en-economia-espanola.pdf

KPMG. (2018, septiembre). Anuario de la restauración organizada en España. https://assets.kpmg/content/dam/kpmg/es/pdf/2018/09/anuario-restauracion-organizada-espana.pdf

Makro. (2020,1 de abril). Impacto del COVID-19 en el sector de la hostelería. https://www.unopuntosiete.es/impacto\%20coronavirus\%20en\%20la\%20hosteleria.pdf

Marín, A. (2020,12 de abril). El 20 por ciento de los restaurantes van a desaparecer. El País. https://elpais.com/elpais/2020/04/11/icon/1586602600_831005.html

Mesa Editorial. (2020, 28 de abril). De la Riva lanza Zona de Reconexión. Merca 2.o. https://www.merca20.com/de-la-riva-lanza-zona-de-reconexion/

Moles, A. (1995). Las ciencias de la impreciso. Miguel Ángel Porrúa, Universidad Autónoma Metropolitana Azcapotzalco.

Morris, C. W. (1946). Signs, Language and Behavior. Prentice Hall.

Nieto, C. (1997). La conciencia lingüística de la filosofía. Ensayo de una crítica de la razón lingüística. Ensayo de una critica de la razón lingüística. Trotta, Fundación Marcelino Botín.

Nosnik, A. (2001). Comunicadores y Organizadores. Planeación y gestión efectiva de su desempeño. 
Nosnik, A. (2012). Teoría de la Comunicación Productiva. HomosSapiens.

oms. (2020). Cómo se transmite la COVID-19. https://www.who.int/es/emergencies/diseases/novel-coronavirus-2019/coronavirus-disease-answers?query=formas $+\mathrm{de}+$ contagio + del+covid 19

Ordine, N. (2020, 11 de abril). Edgar Morin: "Vivimos en un mercado planetario que no ha sabido suscitar fraternidad entre los pueblos". El País.

Público, d. (2020, 1 de junio). cartulinasfluorescentes. https://www.instagram.com/p/ CBZWgkZjSAr/

Piaget, J. (1975). Introducción a la epistemología genética. Paidós.

Postman, N. (1991). Divertirse hasta morir. Ediciones de la Tempestad e Ideas.

Redacción. (2020,17 de junio). “De corazón, muchas gracias”, restaurante cierra y así agradece a sus clientes.

Redacción BBC-Mundo. (2020, 16 de marzo). News Mundo. https://www.bbc.com/mundo/noticias-51912089

Rivera, E. (2010). La construcción del problema de investigación en estudiantes de Ciencias de la Comunicación [tesis inédita de doctorado]. Universidad Nacional Autónoma de México.

Rivera, E. (2019). Transformación del Consumidor: Percepción, autopercepción y microsegmentación; hacia una herramienta de tipificación en marketing y publicidad. 1er Encuentro Nacional de la Red Iberoamericana de Investigadores en Publicidad "Repensar la publicidad y la mercadotecnia: nuevos modelos, nuevos lenguajes". Universidad Anáhuac México.

Scruton, R. (2010). Usos del pesimismo. El peligro de la falsa esperanza. Ariel.

Sánchez de la Nieta, M., \& Fuente, C. (2020). Periodismo vs desinformación: la función social del periodista profesional en la era de las fake news y la posverdad. Sintaxis, $1(4), 1-18$.

Sagan, C. (1986). Contacto. Plaza \& Janés.

Secretaría de Turismo. (2018). Gastronomía, pilar del patrimonio cultural y la economía mexicana:Sectur.https://www.gob.mx/sectur/prensa/gastronomia-pilar-del-patrimonio-cultural-y-la-economia-mexicana-sectur\#: :text=La\%20gastronom\%C3\%ADa\%20 es $\% 20$ una $\% 20$ expresi $\%$ C3\%B3n,la\%20Rep\%C3\%BAblica $\% 2 C \% 20$ Salvador $\% 20$ S\%C3\%A1nchez\%20Estrada

Shannon, C. E., \& Weaver, W. (1948). The Mathematical Theory of Communication. University of Illinois Press.

Signorelli, A. (1999). Antropología urbana. Anthropos, Universidad Autónoma Metropolitana Iztapalapa.

Simetría. (2020, 6 de mayo). El coronavirus y la brecha digital. Animal Político.

The Worlds 50 best. (2020). The World's 50 Best Restaurants. https://www.theworlds50best.com/list/1-50 
Unesco. (2010). La cocina tradicional mexicana, cultura comunitaria, ancestral y viva - El paradigma de Michoacán. https://ich.unesco.org/es/RL/la-cocina-tradicional-mexicana-cultura-comunitaria-ancestral-y-viva-el-paradigma-de-michoacan-00400

Velásquez, A. R. (2018). De los mass media a los medios sociales: reflexiones sobre la nueva ecología de los medios. Revista Latina de Comunicación Social, (73), 583-594.

Watzlawick, P., Bavelas, J. B., \& Jackson, D. D. (1991). Teoría de la Comunicación Humana. Herder.

Woldenberg, J. (2020, 21 de abril). Chava Flores: la esquina de mi barrio. El Universal. 